\title{
Dynamics of self-organized driven particles with competing range interaction
}

\author{
H. J. Zhao, V. R. Misko, and F. M. Peeters \\ Department of Physics, University of Antwerpen, Groenenborgerlaan 171, B-2020 Antwerpen, Belgium
}

(Received 18 November 2012; revised manuscript received 22 February 2013; published 19 August 2013)

\begin{abstract}
Nonequilibrium self-organized patterns formed by particles interacting through competing range interaction are driven over a substrate by an external force. We show that, with increasing driving force, the preexisted static patterns evolve into dynamic patterns either via disordered phase or depinned patterns or via the formation of nonequilibrium stripes. Strikingly, the stripes are formed either in the direction of the driving force or in the transverse direction, depending on the pinning strength. The revealed dynamical patterns are summarized in a dynamical phase diagram.
\end{abstract}

DOI: 10.1103/PhysRevE.88.022914

PACS number(s): 89.75.Kd, 82.70.Dd, 74.25.Uv

\section{INTRODUCTION}

Most of the studies on self-assembly focused on equilibrium systems which form highly ordered arrays (crystals). Equilibrium systems with a competing repulsive and attractive interaction [1-6] demonstrate a variety of morphologies with an inhomogeneous distribution of particles, including stripes, clusters, bubbles, etc. However, most important are dynamic self-assembling systems, i.e., out-of-equilibrium systems that form their characteristic order when dissipating energy $[7,8]$. Understanding and controlling such systems are required in order to maximize the value of self-assembly as a strategy for synthesis and fabrication and, e.g., for the understanding of the role of self-assembly in biology.

Interacting systems driven on a substrate include, e.g., colloids [9-12], charge density waves [13,14], Wigner crystals [15], and vortex matter in superconductors [16-27]. Thus, in a two-dimensional (2D) colloidal crystal driven across ordered substrates, kinks and antikinks were experimentally observed [12]. For vortex matter driven on a random substrate, various dynamical regimes: pinned, plastic, and elastic flows were revealed in experiments [16-20] and in theory [21-27]. Using a periodic substrate instead of random results in rich dynamics including negative differential resistivity of either $N$ type [28] or $N$ and $S$ types [29,30]. Furthermore, driven vortex matter on quasiperiodic substrates [31-35] revealed electronlike or holelike "vortex conductivity" [36]. In the above examples, the interaction between the fundamental units (i.e., colloids or vortices) was purely repulsive with a single length scale.

In the case of competing interaction characterized by two (or more) length scales, systems self-organize forming equilibrium or, at least, metastable patterns. The main question is, how the preexisted static patterns will evolve being driven on a random or regular substrate? And, will they evolve in some other dynamically induced nonequilibrium patterns which do not exist in equilibrium? These are still important open questions for the general case of competing interaction and for an arbitrary potential-energy profile of the substrate. Only a few studies have been performed for some special cases. For example, the dynamical reordering in the plastic regime $[2,3,37,38]$ was examined in a system with competing short- and long-range interactions [2,3].

In the previous studies, the interaction at long distances was repulsive since an attractive interaction leads to phase separation. However, as shown recently [6], a competing interaction with a repulsive core and a fast decaying attractive tail can induce long-living metastable states. This model [6] is applicable to a number of diverse physical systems with fast decaying interaction, e.g., atoms and molecules (Lennard-Jones), colloids, and ferrofluids. Also, it can model intervortex interaction in low- $\kappa[37,39,40]$ and multiband superconductors, such as $\mathrm{MgB}_{2}$ where inhomogeneous vortex patterns, e.g., gossamers and stripes, were recently revealed experimentally $[41,42]$. Those unusual patterns result from a nonmonotonic intervortex interaction as argued in Refs. [43-49]. Furthermore, recent scanning Hall probe microscopy measurements [50] revealed stripe vortex patterns in $\mathrm{MgB}_{2}$ which were not due to inhomogeneous pinning but rather suggestive of another source of ordering, e.g., an external driving bias that might orient the stripes. Therefore, studying the dynamics of systems with competing range interaction is of both fundamental interest and useful for the understanding of unusual experimental patterns.

\section{MODEL}

We study the dynamics of particles with a nonmonotonic interaction moving in $2 \mathrm{D}$ by numerically integrating the Langevin equations using molecular-dynamics simulations. The overdamped equation of motion is $[2,3,6,51,52]$ as follows:

$$
\eta \mathbf{v}_{i}=\mathbf{F}_{i}=\sum_{j \neq i} \mathbf{F}_{i j}+\mathbf{F}_{i}^{p}+\mathbf{F}_{i}^{T}+\mathbf{f}_{d}
$$

We follow the model introduced in Refs. [6,52] and used in Ref. [53] where the interparticle interaction $\mathbf{F}_{i j}$ has a repulsive core and an attractive tail,

$$
\mathbf{F}_{i j}=F_{0}\left(\frac{K_{1}\left(r_{c} / \lambda\right)}{K_{1}\left(b r_{c} / \lambda\right)} K_{1}\left(b r_{i j} / \lambda\right)-K_{1}\left(r_{i j} / \lambda\right)\right) \hat{\mathbf{r}}_{i j},
$$

where $K_{1}$ is the first-order modified Bessel function and $F_{0}(\lambda)$ is the unit of force (length). $\mathbf{F}_{i}^{p}$ is the interaction of the particles with pinning sites,

$$
\mathbf{F}_{i}^{p}=\sum_{k}^{N_{p}}\left(f_{p} / r_{p}\right)\left|\mathbf{r}_{i}-\mathbf{r}_{k}^{(p)}\right| \Theta\left(r_{p}-\left|\mathbf{r}_{i}-\mathbf{r}_{k}^{(p)}\right| / \lambda\right) \hat{\mathbf{r}}_{i k}^{(p)} .
$$

Here $N_{p}$ is the number of pinning sites, $f_{p}$ is the maximum pinning force, $r_{p}$ is the pinning range, $\Theta$ is the Heaviside step function, and $\hat{\mathbf{r}}_{i k}^{(p)}=\left(\mathbf{r}_{i}-\mathbf{r}_{k}^{(p)}\right) /\left|\mathbf{r}_{i}-\mathbf{r}_{k}^{(p)}\right| . \mathbf{F}_{i}^{T}$ is the thermal 
stochastic force, which obeys the following conditions:

$$
\left\langle F_{i}^{T}(t)\right\rangle=0,
$$

and

$$
\left\langle F_{i}^{T}(t) F_{j}^{T}\left(t^{\prime}\right)\right\rangle=2 \eta k_{B} T \delta_{i j} \delta\left(t-t^{\prime}\right) .
$$

The last term $f_{d}$ in Eq. (1) is the applied driving force. We consider a 2D square simulation box $L_{x} \times L_{y}$ (where $L_{x}=$ $L_{y}=L=120$ ) in the $x y$ plane and apply periodic boundary conditions [54]. For the interaction force given by Eq. (2), which decays exponentially for large $r$, we use a cutoff [55] $r=8$. Thus, the interaction in our model is finite range. The initial states are obtained by performing simulated annealing simulations of interacting particles without driving. Then, the driving force is turned on at zero temperature [56].

\section{DYNAMICAL REGIMES}

The dynamics of the system is determined by three main factors: the interparticle, particle-pinning interactions, and the applied driving. To characterize the dynamical regimes, we calculate the average velocity $\langle v\rangle$ versus $f_{d}$ for varying $f_{p}$. In general, for very weak $f_{d}$, most of the particles are pinned resulting in $\langle v\rangle \gtrsim 0$. In the opposite limit of large $f_{d}\left(>f_{p}\right)$, all the particles are depinned, and $\langle v\rangle \propto f_{d}$. The intermediate range of $f_{d}\left(0<f_{d}<f_{p}\right)$ exhibits rich dynamics. Depending on $f_{p},\langle v\rangle-f_{d}$ curves acquire welldistinguished parts (Fig. 1) indicative of different dynamical regimes. In the Supplemental Material [57], examples of snapshots are presented illustrating various dynamical regimes and transitions (crossovers) between them.

Below we present our results for specific pinning regimes and the following interaction parameters: $r_{c}=2.4$ and $b=$ 1.1 , the particle density $n=N / L^{2}=0.0139$, where $N=$ 2000 is the total number of particles in the simulation cell, and the density of random pinning $n_{p}=n$. Note that static configurations for these parameters are labyrinths [6].

\section{A. Weak pinning}

Very weak pinning with strength $\left(f_{p} \sim 0.1\right)$, which is comparable to the elastic force due to the interparticle interaction, is insufficient to destroy the static morphology. Therefore, for weak driving $\left(f_{d}<0.03\right)$, particles only flow inside the labyrinths [Fig. 2(a)] (these are unpinned particles which are trapped by the pinned neighbors, i.e., "collectively
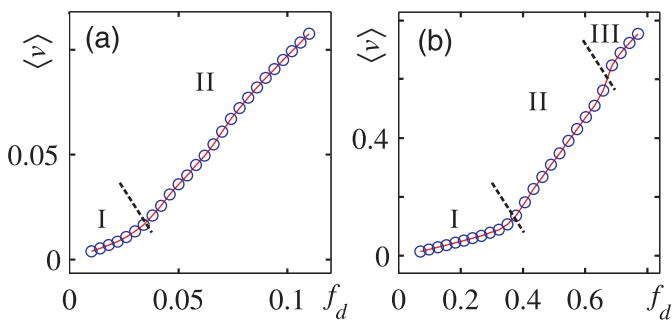

FIG. 1. (Color online) The average velocity $\langle v\rangle$ versus the driving force $f_{d}$ for different strengths of pinning force (a) $f_{p}=0.1$ and (b) $f_{p}=0.7$. For weak pinning, there are two dynamical regimes. For stronger pinning (b), the curve has an "S-like" shape, which exhibits three dynamical regimes.
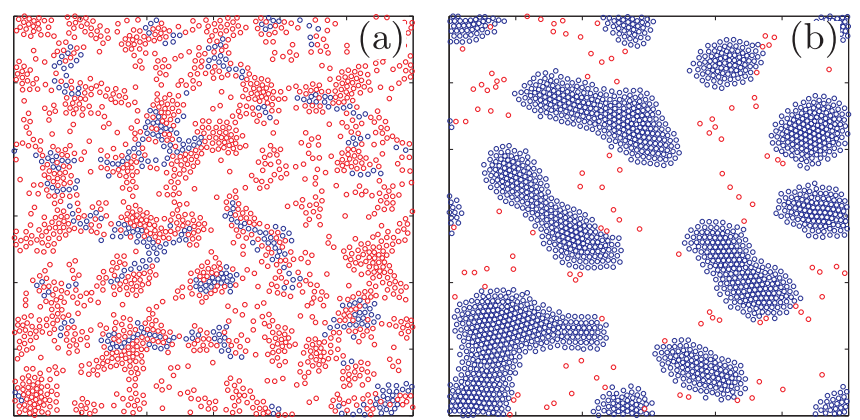

FIG. 2. (Color online) Dynamical patterns for $f_{p}=0.1$, (a) $f_{d}=$ 0.014 , and (b) $f_{d}=0.07$. The mobile (pinned) particles are shown by blue (red) dots. As $f_{d}$ increases, the dynamical pattern changes from (a) partially pinned labyrinths to (b) moving clusters.

pinned") resulting in very small $\langle v\rangle$ [Fig. 1(a)]. For $f_{d}$ larger than some threshold (e.g., $f_{d}=0.038$ ), most of the particles depin, although $f_{d} \ll f_{p}$. This collective depinning occurs as follows: Parts of the labyrinths depin and form small clusters which repin again if driving is weak. For stronger driving, they merge with pinned parts and depin them, leaving only a few individually pinned particles [see Fig. 2(b)]. As a result, the number of mobile particles increases with time, until most of them are depinned.

\section{The optimal cluster size}

Merging of small clusters is not the only mechanism for increasing the cluster size. Strikingly, they can also "grow" by collecting individual particles generated by "evaporating" small clusters.

To understand this effect, we explore the analogy between a cluster moving on a pinning landscape and a droplet of water moving on a prewetted substrate [58,59]. The size of the droplet increases if the substrate is overwetted and vice versa: The size of the droplet decreases if the substrate is dry. Therefore, there exists a critical wettability when the droplet keeps its size. Translating this to the language of our system, larger clusters have larger attraction to their tail particles. Therefore, the critical wettability of large clusters is smaller than that of small ones. Now, considering a small cluster following a large one, the substrate passed by the large cluster is always dry for the small cluster. Thus, the small cluster evaporates and leaves an overwetted substrate for the large clusters.

Figure 3 illustrates the evolution of a small cluster following a large cluster. The small cluster remains stable until the cluster in front of it becomes large enough [Fig. 3(a)]. Then the small cluster gradually loses its particles [see Fig. 3(b)] and finally disappears. As a result, a steady state is formed when all the clusters are large and are well separated.

\section{B. Intermediate pinning}

For the pinning force $f_{p}$ larger than the strength of the typical interparticle interaction force $\left(0.3 \leqslant f_{p} \leqslant 0.7\right)$, the preexisted patterns are destroyed. There are three dynamical regimes in the case of intermediate pinning strength [Fig. 1(b)]. In regime I, the pinning force is larger than the elastic force due to the interparticle interaction, whereas, the driving force 

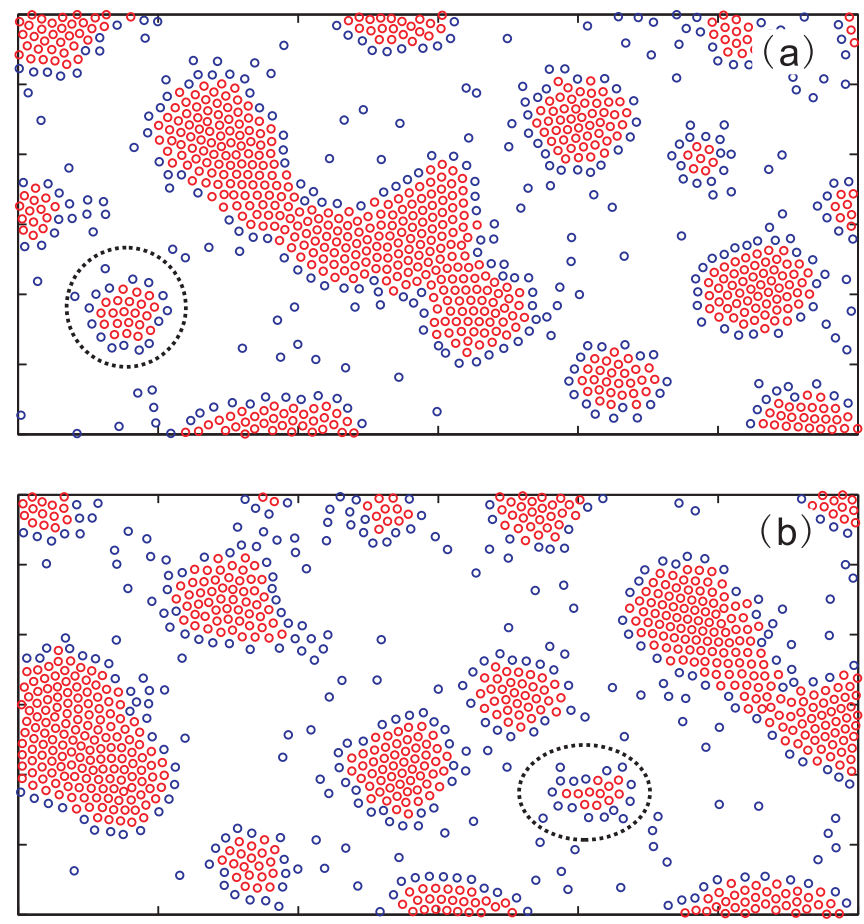

FIG. 3. (Color online) Evaporation of a small cluster (marked by the black dotted lines) following a large cluster (moving from the left to the right) for $f_{d}=0.062, f_{p}=0.1$, (a) $t=1900 \Delta t$, and (b) $2500 \Delta t$.

is smaller. Therefore, part of the labyrinths can still exist, i.e., in the form of deformed small clusters.

A stronger driving force $f_{d}$ can overcome the elastic force between particles and the pinning force, resulting in more mobile particles, which increases $\langle v\rangle$. A further increase in $f_{d}$ increases the density of mobile particles, until all the particles are depinned (regime II). In turn, this regime can be divided into three subregimes: (i) $f_{d}$ is just above the elastic force: The resulted dynamical patterns are disordered [Fig. 4(a)]; (ii) stronger $f_{d}$ : The mobile particles form clusters [Fig. 4(b)], which elongate in the direction of driving (i.e., for $r_{c}>2.3$, see Ref. [6]) when the "head" and tail of a cluster move with different velocities because of the different "frictions" due to the pinning (note that the motion in the medium, without pinning, is overdamped, whereas, the additional friction is related to the pinning); (iii) even larger $f_{d}$ : The elongated clusters connect to each other, which restores a continuous particle flow [Fig. 4(c)].

Although the stripe formation has been found in many physical systems, e.g., driven vortices [6,37,40,52], 2D electrons [2,3,51], and systems with shoulder potential [60], the stripe formation and orientation in our case are different. In those studies, the interparticle interaction for long distances was repulsive, which prevented the stripes from merging and arranged them parallel. In contrast, here the weak attractive tail leads to the accumulation of single mobile particles but is not sufficient for merging the stripes locked by the random pinning. As a consequence, the stripes have varying widths and interstripe distances in contrast to the ones resulting from a purely repulsive tail [2].
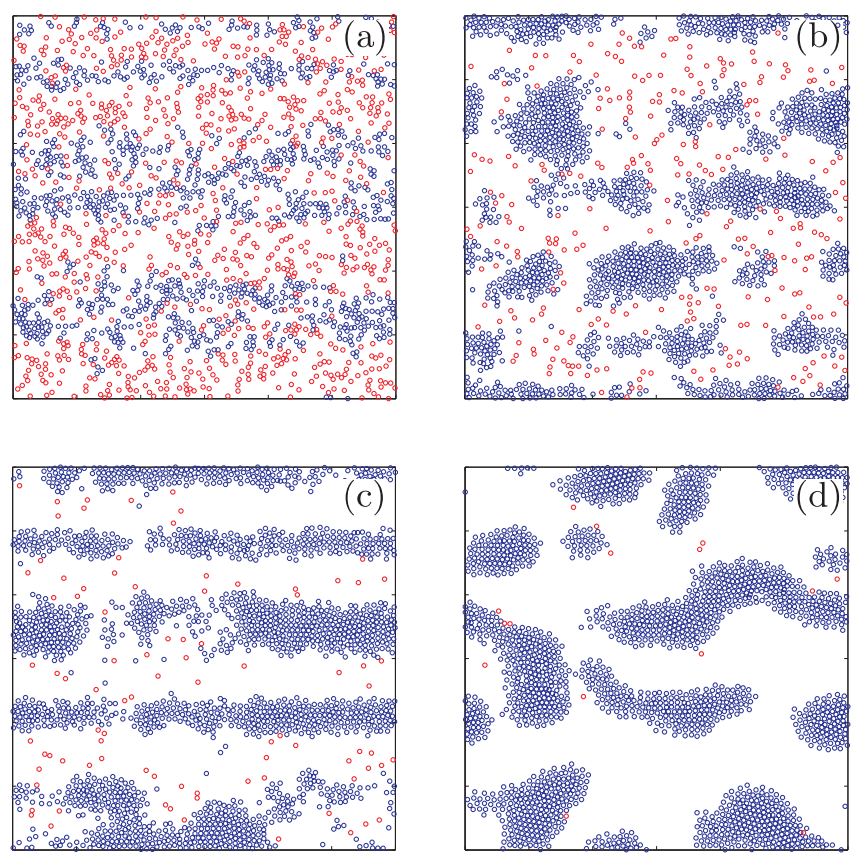

FIG. 4. (Color online) Dynamical patterns for $f_{p}=0.5$, (a) $f_{d}=$ 0.21 , (b) $f_{d}=0.39$, (c) $f_{d}=0.43$, and (d) $f_{d}=0.49$. Blue (red) dots stand for mobile (pinned) particles.

Finally, for $f_{d}>f_{p}$, all the particles are depinned, the clusters or stripes are not oriented [Fig. 4(d)], and $\langle v\rangle$ increases linearly versus $f_{d}$ (regime III) [28-30].

\section{Strong pinning: Formation of transverse stripes}

For sufficiently strong pinning $\left(f_{p} \geqslant 0.9\right)$, even a small number of pinning sites destroy the pattern structure. As a result, for a broad range of $f_{d}$, the dynamical patterns are disordered. Moving clusters are formed when $f_{d}$ is very close to the depinning threshold: $f_{d} \lesssim f_{p}$.

For the so-called "fast sliding regime," when $f_{d}>f_{p}$, the random pinning has the same impact on the moving patterns as the thermal Langevin force [21]. Strong pinning orients the sliding triangular lattice in the longitudinal direction [Fig. 5(a)], i.e., particles move in static channels, which were found for moving elastic lattices with disorder [23]. Once these channels are formed, there are energy barriers for transverse motion, i.e., the motion along the longitudinal channels is
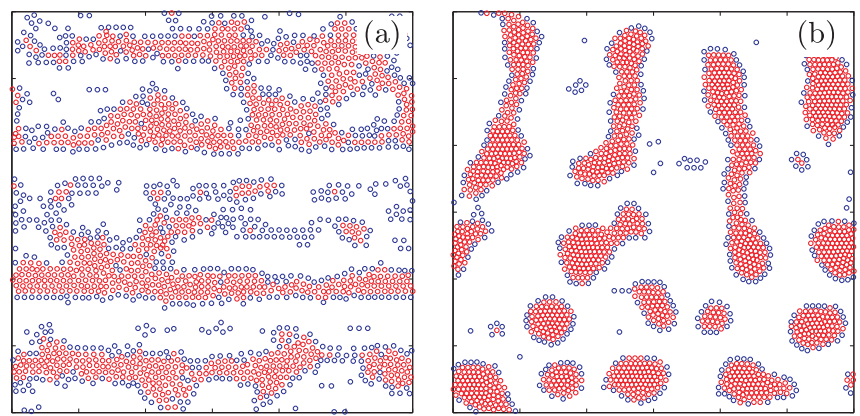

FIG. 5. (Color online) Stripes in fast sliding dynamical regimes. (a) Longitudinal stripes for $r_{c}=2.5, f_{p}=1.7$, and $f_{d}=1.8275$ and (b) transverse stripes for $r_{c}=2.3, f_{p}=1.7$, and $f_{d}=2.465$. 


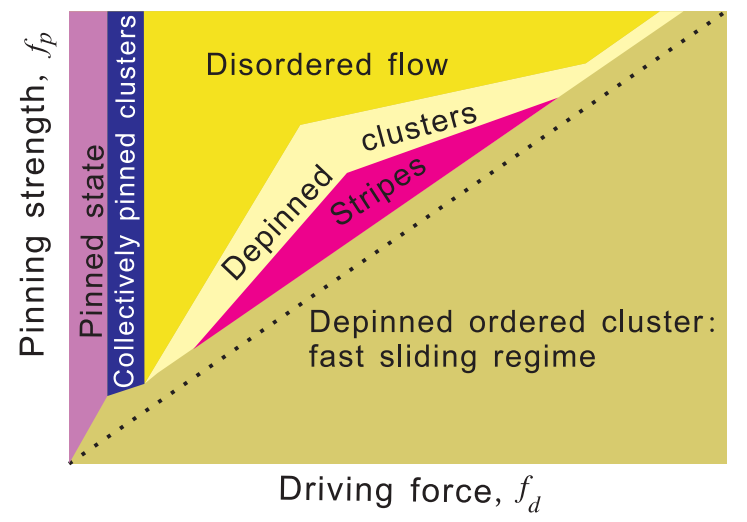

FIG. 6. (Color online) Schematic dynamical phase diagram in plane $f_{p}$ versus $f_{d}$.

locked. These transverse energy barriers can be overcome by a weak bias force, e.g., a weak external driving in the transverse direction.

We use the paradigm of static channels to explain a very unusual behavior revealed for $r_{c} \lesssim 2.3$ : the formation of striking transversal stripes [see Fig. 5(b)]. To understand this unusual behavior, recall that, in the absence of pinning, particles try to form, due to the attraction force, circular clusters for this value of $r_{c}$ [6]. The aggregation due to the inter-particle attraction, in principle, overcomes the elongation of clusters due to the formation of static channels. However, the latter process is much faster than the former one, and as a result, for strong enough driving, the clusters turn out to be dynamically locked by the static channels. Merging clusters still tend to acquire circular shape, but due to the existing transverse barriers, they become "stretched" along the transverse direction, thus, resulting in the observed transverse stripes [Fig. 5(b)].

\section{PHASE DIAGRAM}

The dynamical phase diagram in the plane of "pinning strength $f_{p}$ versus driving force $f_{d}$ " is shown in Fig. 6. The pinned state is formed when the driving force is extremely small, i.e., insufficient to destroy the patterns. The depinned state corresponds to large driving forces. Note that, due to collective depinning, assisted by the elastic force between the particles, the actual threshold is smaller than the maximum pinning force (dashed line). For weak pinning, there are two dynamical regimes: pinned clusters (labyrinths) with particle flow inside the labyrinth [Fig. 2(a)] and depinned clusters
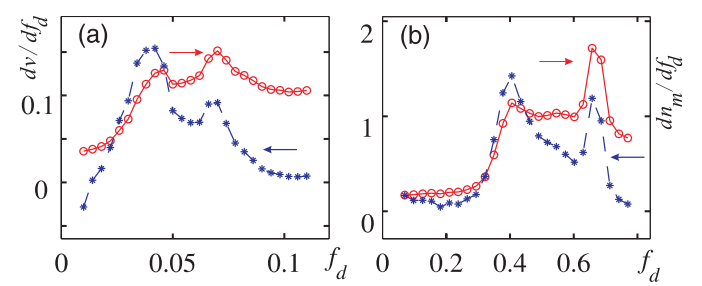

FIG. 7. (Color online) The derivatives of the average velocity $d v / d f_{d}$ (solid red line and circles) and of the number of mobile particles $d n / d f$ (dashed blue line and stars) versus $f_{d}$ for (a) $f_{p}=0.3$ and (b) $f_{p}=0.9$.
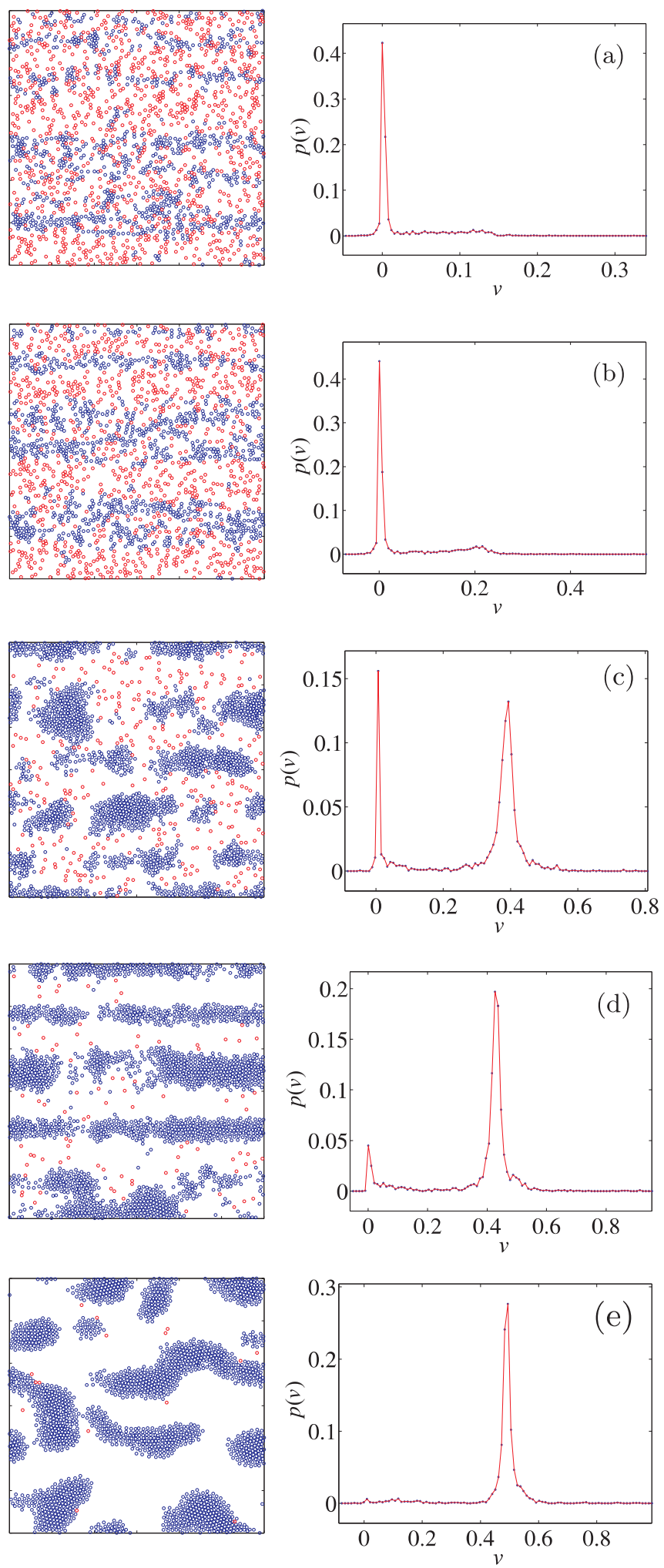

FIG. 8. (Color online) Left panel: Dynamical patterns for pinning strength $f_{p}=0.5$ and driving force (a) $f_{d}=0.13$, (b) $f_{d}=0.21$, (c) $f_{d}=0.39$, (d) $f_{d}=0.43$, and (e) $f_{d}=0.49$. The mobile particles are shown by blue (gray) dots, whereas, the pinned or collectively pinned particles are shown by red (black) dots. Right panel: Corresponding velocity distributions $p(v)$.

[Fig. 2(b)] or stripes. For intermediate pinning, we distinguish: (i) "pinned clusters with chainlike flows;" (ii) "intermediate 
regime" including chainlike flows with a background of disordered pinned particles [Fig. 4(a)], reordered depinned clusters [Fig. 4(b)], and oriented stripes [Fig. 4(c)]; and (iii) "depinned elastic flow" [Fig. 4(d)]. With increasing $f_{p}$, the driving range for the formation of depinned clusters and stripes becomes very narrow.

\section{A. Peak effect}

To analyze the curvature of the $\langle v\rangle-f_{d}$ curve (Fig. 1), we take the derivative $d\langle v\rangle / d f_{d}$ and find that each curve displays two main peaks (Fig. 7). With increasing $f_{d}$, there are two contributions to $\langle v\rangle$ : the varying number of mobile particles and the increasing particle velocity. The nonlinear behavior of $d\langle v\rangle / d f_{d}$ is due to the former contribution. The variation in the number of mobile particles $n_{m}=v / f_{d}$ is as follows: $d n_{m} / d f_{d}=\left(d v / d f_{d}\right) f_{d}^{-1}-v f_{d}^{-2}$. As shown in Fig. 7, $d n_{m} / d f_{d}$ also has two peaks, which are related to the peaks in $d v / d f$. The two-peak structure in $d n_{m} / d f_{d}$ (and $d\langle v\rangle / d f_{d}$ ) is explained as follows. The first peak $\left(f_{d} \approx 0.5 f_{p}\right)$ : When $f_{d}$ increases, it depins more particles resulting in the growth of $d n_{m}$, then the number of pinned particles decreases. The second peak appears when $f_{d}$ reaches the depinning threshold.

The distinct dynamical patterns can also be characterized by other "dynamical order parameters" (for static patterns, see Ref. [6]). The transitions (crossovers) between the dynamical patterns are observed as features in these order parameters, such as the velocity distribution and the surface-area-tovolume ratio. (Note that the transitions between the different dynamical phases are rather smooth, although the different
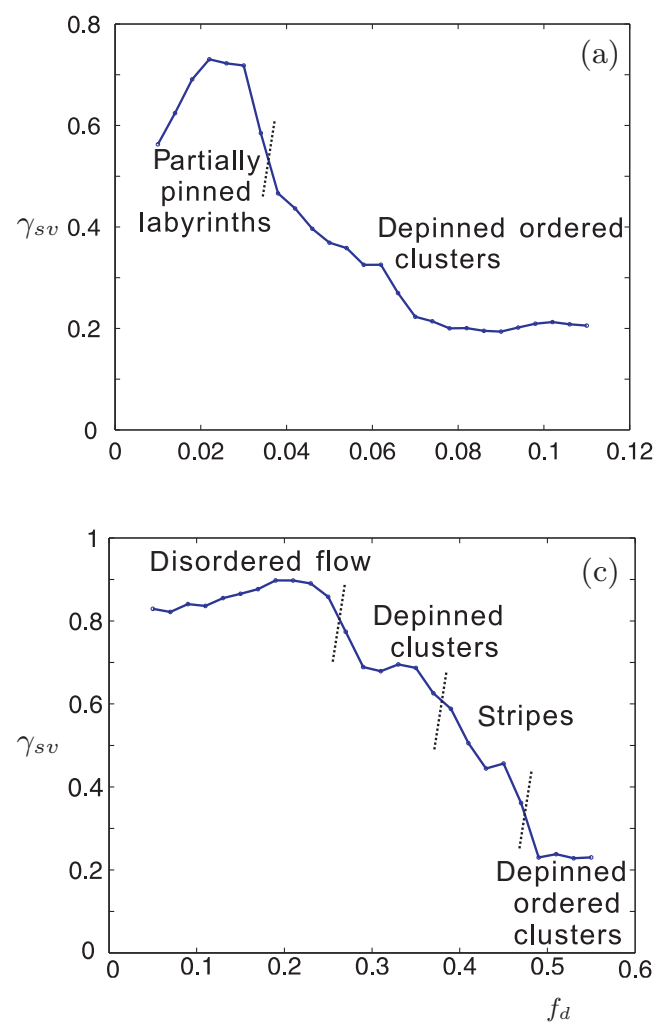

phases are well distinguished as peaks in the corresponding order parameters.)

\section{B. Velocity distribution}

In Fig. 8, we plot the dynamical patterns and the corresponding velocity distributions for pinning strength $f_{p}=0.5$. For the driving force $f_{d}<0.21$ [see Fig. 8(a)], there is only one peak positioned at 0 in the plot "probability $p(v)$ versus velocity $v$." This corresponds to the state when all particles are pinned.

The second peak, which starts to develop as a weak feature at applied driving $f_{d} \geqslant 0.21$, corresponds to the situation when the mobilized (depinned) particles start to form clusters [see Fig. 8(b)]. Further increasing $f_{d}$ clearly leads to the formation of clusters, which strengthens the second peak. Simultaneously, the first peak weakens [see Fig. 8(c)]. The two-peak structure shown in Fig. 8(c) corresponds to moving clusters (second peak) and pinned ones (first peak).

Stripes are formed when the second peak is strong enough, i.e., enough mobilized (unpinned) particles are generated to form stripes [see Fig. 8(d)]. Finally, when $f_{d}>f_{p}$, all the particles are mobilized. As a result, the first peak disappears, and the second peak shifts towards higher values indicating that all the particles participate in the motion with the same velocity.

\section{Surface-area-to-volume ratio}

In Fig. 9, we plot "surface-area-to-volume ratio $\gamma_{s v}$ versus driving force $f_{d}$." (Note that the state with all the particles
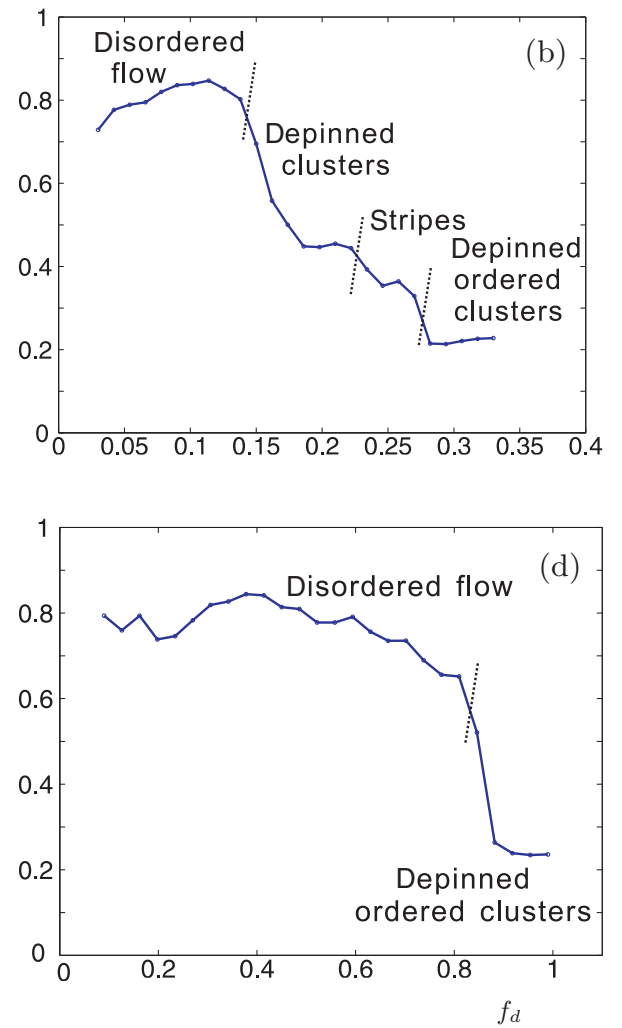

FIG. 9. (Color online) Surface-area-to-volume ratio $\gamma_{s v}$ versus driving force $f_{d}$ for (a) $f_{p}=0.1$, (b) $f_{p}=0.3$, (c) $f_{p}=0.5$, and (d) $f_{p}=0.9$. 
pinned is not considered in this section.) For weak pinning $\left(f_{p}=0.1\right)$, there are only two dynamical regimes as discussed in the main text [see Fig. 9(a)]. As $f_{d}$ increases, $\gamma_{s v}$ first increases since the applied driving destroys the preexisted static patterns, i.e., labyrinths or clusters. The fast decrease in the function $\gamma_{s v}$ corresponds to the onset of the collective depinning of clusters. The saturation region for $f_{d} \geqslant 0.074<f_{p}$ indicates that the threshold of collective depinning in this case is much lower than the pinning force.

For intermediate pinning $\left(0.3 \leqslant f_{p} \leqslant 0.7\right)$, similar to the weak pinning case, $\gamma_{s v}$ first increases when increasing $f_{d}$ [see Figs. 9(b) and 9(c)]. Then $\gamma_{s v}$ rapidly decreases when mobilized particles start to cluster. A further increase in $f_{d}$ results, on one hand, in the depinning of additional individual particles (they are counted as "surface particles"). On the other hand, small clusters still can survive for these values of driving force $f_{d}$ (smaller clusters correspond to larger $\gamma_{s v}$ ). As a result of the competition of these two effects, a weak plateau [see Figs. 9(b) and 9(c)] appears in $\gamma_{s v}$. The latter effect disappears when all small clusters merge into stripes, thus, $\gamma_{s v}$ start to rapidly decrease again. A fast drop in $\gamma_{s v}$ appears when $f_{d}>f_{p}$. The reason is that, in this case, the particles form large clusters which have much lower $\gamma_{s v}$ than stripes.

For the strong pinning case $\left(f_{p} \geqslant 0.9\right)$, the dynamical pattern changes directly from a disordered flow to depinned ordered clusters. Thus, a fast decay in $\gamma_{s v}$ is observed when $f_{d}$ is close to $f_{p}$.

Note that, for all the values of pinning $f_{p}$ considered above, the function $\gamma_{s v}$ has a constant minimum value $(\approx 0.2)$ when $f_{d}>f_{p}$, which indicates that they all share similar states (depinned ordered clusters).

\section{CONCLUSIONS}

Analyzing the dynamics of patterns formed due to a short-range nonmonotonic interaction, we found, depending on the strength of the pinning, a variety of dynamical regimes including: a disordered motion (when the preexisted static patterns are destroyed), the motion of depinned clusters, and the formation of stripes. Strikingly, the stripes can be either longitudinal (i.e., formed in the direction of the driving force) or transverse (i.e., formed in the direction normal to the driving), depending on the pinning regime: weak or strong, correspondingly. We also found that, unlike in the previously studied static case, the motion on a random substrate dynamically selects the optimal cluster size. Clusters smaller than this size lose their particles due to the effect of friction and finally evaporate, whereas, large clusters collect these individual particles and grow until only large enough clusters remain in the system. The mechanism of this dynamical size selection effect is similar to the critical wettability of a water droplet moving on either an overwetted or a dry substrate. We found a pronounced double-peak structure in the variation of the $\langle v\rangle-f_{d}$ curve, which is explained by: (i) increasing the number of depinned particles and the simultaneous decrease in the number of pinned particles which can be depinned and (ii) approaching the depinning threshold for individual particles.

Our findings can be useful for the analysis of dynamical pattern formation in various systems with competing range interactions, including colloids, vortices in superconductors, etc., as well as for the deeper understanding of wetting phenomena and microscopic friction. In particular, we believe that the dynamical patterns, predicted in our paper, can be verified in experiments with driven colloids and with driven vortices in two-band superconductors, such as $\mathrm{MgB}_{2}$.

\section{ACKNOWLEDGMENTS}

This work was supported by the "Odysseus" Program of the Flemish Government and the Flemish Science Foundation (FWO-Vl).
[1] M. Seul and D. Andelman, Science 267, 476 (1995).

[2] C. Reichhardt, C. J. Olson, I. Martin, and A. R. Bishop, Europhys. Lett. 61, 221 (2003).

[3] C. Reichhardt, C. J. Olson Reichhardt, I. Martin, and A. R. Bishop, Phys. Rev. Lett. 90, 026401 (2003).

[4] K. Nelissen, B. Partoens, and F. M. Peeters, Phys. Rev. E 71, 066204 (2005)

[5] E. Y. Vedmedenko, Competing Interactions and Patterns in Nanoworld (Wiley-VCH Verlag GmbH, Weinheim, 2007).

[6] H. J. Zhao, V. R. Misko, and F. M. Peeters, New J. Phys. 14, 063032 (2012).

[7] G. Whitesides and M. Boncheva, Proc. Natl. Acad. Sci. USA 99, 4769 (2002).

[8] P. Ball, The Self-Made Tapestry: Pattern Formation in Nature (Oxford University Press, Oxford, 1999).

[9] C. Reichhardt and C. J. Olson, Phys. Rev. Lett. 89, 078301 (2002).

[10] Y. Cao, J. Chen, Y. Wang, Z. Jiao, and W. K. Chow, Phys. Rev. B 68, 134209 (2003).
[11] A. Pertsinidis and X. S. Ling, Phys. Rev. Lett. 100, 028303 (2008).

[12] T. Bohlein, J. Mikhael, and C. Bechinger, Nat. Mater. 11, 126 (2012).

[13] G. Grüner, Rev. Mod. Phys. 60, 1129 (1988).

[14] L. Balents and M. P. A. Fisher, Phys. Rev. Lett. 75, 4270 (1995).

[15] C. Reichhardt, C. J. Olson, N. Grønbech-Jensen, and F. Nori, Phys. Rev. Lett. 86, 4354 (2001).

[16] S. Bhattacharya and M. J. Higgins, Phys. Rev. Lett. 70, 2617 (1993).

[17] U. Yaron, Nature (London) 376, 753 (1995).

[18] A. Duarte, E. Fernandez Righi, C. A. Bolle, F. de la Cruz, P. L. Gammel, C. S. Oglesby, E. Bucher, B. Batlogg, and D. J. Bishop, Phys. Rev. B 53, 11336 (1996).

[19] F. Pardo, F. De La Cruz, P. L. Gammel, C. S. Oglesby, E. Bucher, B. Batlogg, and D. J. Bishop, Phys. Rev. Lett. 78, 4633 (1997).

[20] F. Pardo, F. de la Cruz, P. L. Gammel, E. Bucher, and D. J. Bishop, Nature (London) 396, 348 (1998). 
[21] A. E. Koshelev and V. M. Vinokur, Phys. Rev. Lett. 73, 3580 (1994).

[22] K. Moon, R. T. Scalettar, and G. T. Zimányi, Phys. Rev. Lett. 77, 2778 (1996).

[23] T. Giamarchi and P. Le Doussal, Phys. Rev. Lett. 76, 3408 (1996).

[24] L. Balents, M. C. Marchetti, and L. Radzihovsky, Phys. Rev. Lett. 78, 751 (1997).

[25] C. J. Olson, C. Reichhardt, and F. Nori, Phys. Rev. Lett. 81, 3757 (1998).

[26] B. Rosenstein and D. Li, Rev. Mod. Phys. 82, 109 (2010).

[27] W. V. Pogosov, H. J. Zhao, V. R. Misko, and F. M. Peeters, Phys. Rev. B 81, 024513 (2010).

[28] C. Reichhardt, C. J. Olson, and F. Nori, Phys. Rev. Lett. 78, 2648 (1997).

[29] V. R. Misko, S. Savel'ev, A. L. Rakhmanov, and F. Nori, Phys. Rev. Lett. 96, 127004 (2006).

[30] V. R. Misko, S. Savel'ev, A. L. Rakhmanov, and F. Nori, Phys. Rev. B 75, 024509 (2007).

[31] V. Misko, S. Savel'ev, and F. Nori, Phys. Rev. Lett. 95, 177007 (2005).

[32] V. R. Misko, S. Savel'ev, and F. Nori, Phys. Rev. B 74, 024522 (2006).

[33] J. E. Villegas, M. I. Montero, C.-P. Li, and I. K. Schuller, Phys. Rev. Lett. 97, 027002 (2006).

[34] M. Kemmler, C. Gürlich, A. Sterck, H. Pöhler, M. Neuhaus, M. Siegel, R. Kleiner, and D. Koelle, Phys. Rev. Lett. 97, 147003 (2006).

[35] A. V. Silhanek, W. Gillijns, V. V. Moshchalkov, B. Y. Zhu, J. Moonens, and L. H. A. Leunissen, Appl. Phys. Lett. 89, 152507 (2006)

[36] V. R. Misko, D. Bothner, M. Kemmler, R. Kleiner, D. Koelle, F. M. Peeters, and F. Nori, Phys. Rev. B 82, 184512 (2010).

[37] X. B. Xu, H. Fangohr, Z. H. Wang, M. Gu, S. L. Liu, D. Q. Shi, and S. X. Dou, Phys. Rev. B 84, 014515 (2011).

[38] C. J. Olson Reichhardt, C. Reichhardt, and A. R. Bishop, Phys. Rev. E 83, 041501 (2011).

[39] E. Brandt and M. Das, J. Supercond. Novel Magn. 24, 57 (2011).

[40] X. B. Xu, H. Fangohr, S. Y. Ding, F. Zhou, X. N. Xu, Z. H. Wang, M. Gu, D. Q. Shi, and S. X. Dou, Phys. Rev. B 83, 014501 (2011).
[41] V. Moshchalkov, M. Menghini, T. Nishio, Q. H. Chen, A. V. Silhanek, V. H. Dao, L. F. Chibotaru, N. D. Zhigadlo, and J. Karpinski, Phys. Rev. Lett. 102, 117001 (2009).

[42] T. Nishio, V. H. Dao, Q. Chen, L. F. Chibotaru, K. Kadowaki, and V. V. Moshchalkov, Phys. Rev. B 81, 020506 (2010).

[43] E. Babaev and M. Speight, Phys. Rev. B 72, 180502 (2005).

[44] E. Babaev, J. Carlström, and M. Speight, Phys. Rev. Lett. 105, 067003 (2010).

[45] A. Chaves, L. Komendová, M. V. Milošević, J. S. Andrade, Jr., G. A. Farias, and F. M. Peeters, Phys. Rev. B 83, 214523 (2011).

[46] L. Komendová, M. V. Milošević, A. A. Shanenko, and F. M. Peeters, Phys. Rev. B 84, 064522 (2011).

[47] S.-Z. Lin and X. Hu, Phys. Rev. B 84, 214505 (2011).

[48] L. Komendová, Y. Chen, A. A. Shanenko, M. V. Milošević, and F. M. Peeters, Phys. Rev. Lett. 108, 207002 (2012).

[49] M. Silaev and E. Babaev, Phys. Rev. B 85, 134514 (2012).

[50] J. Gutierrez, B. Raes, A. V. Silhanek, L. J. Li, N. D. Zhigadlo, J. Karpinski, J. Tempere, and V. V. Moshchalkov, Phys. Rev. B 85, 094511 (2012).

[51] C. Reichhardt, C. J. O. Reichhardt, and A. R. Bishop, Europhys. Lett. 72, 444 (2005).

[52] H. J. Zhao, V. R. Misko, and F. M. Peeters, Physica C 479, 130 (2012).

[53] C. Reichhardt, J. Drocco, C. O. Reichhardt, and A. Bishop, Physica C 479, 15 (2012).

[54] To check the effect of boundaries, an enlarged simulation box was used, namely, we tried $L_{x}=L_{y}=L=240$ and $L_{x}=L_{y}=$ $L=360$, which revealed no change in the resulted patterns.

[55] Since both terms of the interaction force are represented by modified Bessel functions, which decay exponentially for large distances, the used cutoff procedure is safe: Our trial simulations with a cutoff of $r>16 \lambda$ did not reveal any impact on the results.

[56] Nonzero temperature would result in the broadening of the particle trajectories and, ultimately, in the destruction of dynamical patterns.

[57] See Supplemental Material at http://link.aps.org/supplemental/ 10.1103/PhysRevE.88.022914 for examples of snapshots illustrating various dynamical regimes and transitions (crossovers) between them for $f_{p}=0.7$ and the varying driving force $f_{d}$.

[58] P. G. de Gennes, Rev. Mod. Phys. 57, 827 (1985).

[59] D. Bonn, J. Eggers, J. Indekeu, J. Meunier, and E. Rolley, Rev. Mod. Phys. 81, 739 (2009).

[60] G. Malescio and G. Pellicane, Nat. Mater. 2, 97 (2003). 